\title{
Homozygosity for a FBN1 missense mutation: clinical and molecular evidence for recessive Marfan syndrome
}

\author{
Bert BA de Vries ${ }^{*, 1}$, Gerard Pals ${ }^{2}$, Roelof Odink ${ }^{3}$ and Ben CJ Hamel ${ }^{1}$ \\ ${ }^{1}$ Department of Human Genetics, Radboud University Nijmegen Medical Centre, Nijmegen, The Netherlands; \\ ${ }^{2}$ Department of Human Genetics, Vrije Universiteit Medisch Centrum, Amsterdam, The Netherlands; ${ }^{3}$ Department of \\ Pediatrics, Catharina Hospital, Eindhoven, The Netherlands
}

Marfan syndrome (MFS) is known as an autosomal-dominant connective tissue disorder (MIM 154700), involving primarily the skeletal, ocular and cardiovascular systems, and caused by mutations in the gene for fibrillin1 (FBN1). Here, we report on two cousins from a consanguineous family with a homozygous c. 1453C $>$ T FBN1 mutation (p.Arg485Cys) and MFS. All four healthy parents were heterozygous for the c. 1453C $>$ T FBN1 mutation and none fulfilled the Ghent criteria for MFS. This family is the first molecularly confirmed recessive MFS. The demonstration of recessive cases of MFS has obvious implications for genetic counselling as well as for molecular diagnosis.

European Journal of Human Genetics (2007) 15, 930-935; doi:10.1038/sj.ejhg.5201865; published online 13 June 2007

Keywords: recessive Marfan syndrome; FBN1 mutation; homozygosity

\section{Introduction}

Marfan syndrome (MFS) is a well-known multisystem connective tissue disorder with an estimated incidence of $\sim 1$ in 5000 individuals. ${ }^{1}$ The pleiotropic manifestations involving primarily the skeletal, ocular, and cardiovascular systems are caused by mutations in the gene for fibrillin1 $(F B N 1){ }^{2}$ The clinical diagnosis is based on the combination of major and minor clinical criteria, known as the Ghent criteria. ${ }^{1,3}$ As mutations in the FBN1 gene have also been found in patients not fulfilling these criteria, MFS should be regarded as one of the type 1 fibrillinopathies. ${ }^{4}$ In the majority of patients with classical MFS, a mutation in the FBN1 gene can be identified with, so far, more than 550 different $F B N 1$ mutations being reported that are

*Correspondence: Dr BBA de Vries, Department of Human Genetics, 849, Radboud University Nijmegen Medical Centre, PO Box 9101, 6500 HB Nijmegen, The Netherlands.

Tel: + 3124 3613946; Fax: + 3124 3668753;

E-mail: b.devries@antrg.umcn.nl

Received 16 October 2006; revised 27 April 2007; accepted 2 May 2007; published online 13 June 2007 spread throughout the gene and mostly unique for each family. ${ }^{5,6}$

The MFS is known as an autosomal-dominant disorder (MIM 154700) and several studies in human and mice have, for a long time, favored a dominant-negative model for the pathogenesis of MFS. ${ }^{7}$ However, recently, based on mice studies a role for a haplo-insuffiency model has been raised, ${ }^{8}$ including the hypothesis that abnormal fibrillin or reduced levels of fibrillin may lead to an excess of TGF- $\beta$ activity. ${ }^{9}$

Here, we report on two cousins from a consanguineous Turkish family with a homozygous c.1453C > T FBN1 mutation (p.Arg485Cys) and MFS.

\section{Materials and methods}

Patients

The parents of both patients, reported in this study, were first cousins and $2 \times 2$ siblings. The pedigree is depicted in Figure 1.

The proband (III.1) was a 22-year-old male born with an intrauterine torsion of the left testis. Neonatally, he had a 


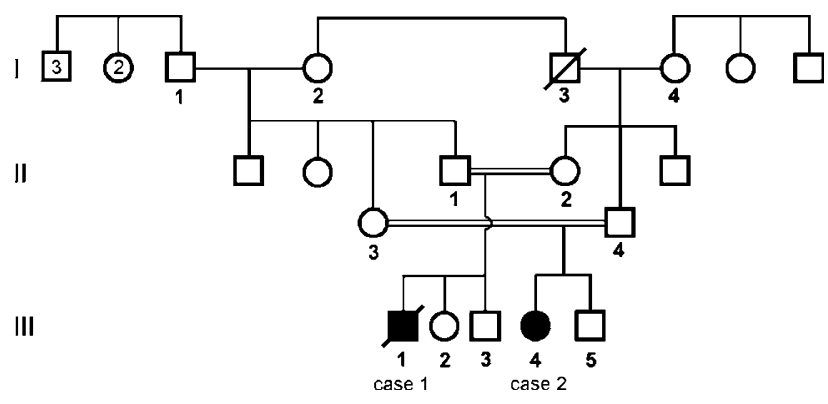

Figure 1 The pedigree with proband (case 1) and his cousin (case 2). Black box indicates affected.

transient primary hypothyroidism for which treatment during 6 months with thyroxine was initiated. At the age of 1 year, a bilateral upward lens subluxation was noted with normal urine homocysteine values. Painful knee problems arose at the age of 13 and in the same period a mild mitral valve prolapse were noted. A year later, he underwent a ptosis correction bilaterally. At 19 years of age, he had a spontaneous right pneumothorax and a year later a dissection of the distal thoracal aorta and the proximal abdominal aorta was diagnosed using MRA. On physical examination at the age of 22 years, normal height of $186 \mathrm{~cm}$ (60th centile), arm span of $188 \mathrm{~cm}$ and head circumference of $58.5 \mathrm{~cm}$ (60th centile) was noted. He had a slight ptosis, prominent nose, a high-arched palate, pectus excavatum, mild scoliosis, long fingers and toes, and mild contractures of the elbows. Thumb and wrist signs were negative; neither striae nor hyperlaxity (Beighton score of 0/9) were noted. At an age of 20 years, he was operated for an aortic valve insufficiency and, 2 years later, he underwent an aorta ascendens replacement because of aortic aneurysm with aortic insufficiency. At the age of 23 years he was found dead. No obduction was permitted by his family. In conclusion, he had MFS according to the Ghent criteria.

His 13-year-old cousin (III.4) was prematurely born after 32 weeks gestation with a birth weight of $2710 \mathrm{~g}$ (97th centile). She had also a transient primary hypothyroidism and treatment for 6 months was initiated. At the age of 3 years, bilateral lens subluxation was noted. Cardiovascular evaluation at the age of nearly 13 years revealed no abnormalities (aortic root diameter, $24 \mathrm{~mm}$ ) and normal urine homocysteine values were measured. On physical examination at the age of 13 years, normal height of $169 \mathrm{~cm}$ (85th centile) arm span of $169 \mathrm{~cm}$, and head circumference of $57 \mathrm{~cm}$ (85th centile) were noted. She had a slight ptosis bilaterally, upward slanting palpebral fissures, high-arched palate and long fingers. Thumb and wrist signs were negative; neither striae nor hyperlaxity (Beighton score of 0/9) were noted. However, a lumbosacral dural ectasia was noted by MRI. At the age of 13 years, this girl has two major criteria and no MFS. As she shares the same FBN1 genotype with her cousin, with the classical Marfan the genetic history can be scored positive. In conclusion, she had MFS according to the Ghent criteria.

The girl's mother (II.3) had an increased span-height ratio (span, $178 \mathrm{~cm}$; height, $166 \mathrm{~cm}$; ratio 1.07 ), higharched palate, and large hands and feet. The male's father (II.1, sib of the girl's mother) had also an increased spanheight ratio (span, $189 \mathrm{~cm}$; height $180 \mathrm{~cm}$; ratio 1.05), high palate, and large hands and feet. For both parents, the wrist and thumbs sign were negative and no other physical Marfan signs were noted. The other parents had normal spanheight ratios: the ratio for girl's father (II.4) 1.02 (span, $186 \mathrm{~cm}$; height $182.5 \mathrm{~cm}$ ) and for the male's mother (II.2) ratio 1.04 (span, $169 \mathrm{~cm}$; height $162.5 \mathrm{~cm}$ ).

Cardiovascular and ophthalmologic evaluation revealed no abnormalities in the four parents. The aortic root diameter of the male's mother (II.2, age 43 years, weight $75 \mathrm{~kg}$ ) was $34 \mathrm{~mm}$ (within 95\% confidence interval (CI) corrected for body surface area (BSA)), of his father (II.1: age 43 years; weight $82 \mathrm{~kg}$ ) was $34 \mathrm{~mm}$ (within 95\% CI corrected for BSA). ${ }^{10}$ The aortic root diameter of the girl's mother (II.3: age 37 years; weight $94 \mathrm{~kg}$ ) was $27 \mathrm{~mm}$ (within 95\% CI corrected for BSA) and her father (II.4: age 40 years; weight $129 \mathrm{~kg}$ ) was $40 \mathrm{~mm}$ (within 95\% CI corrected for BSA).

Further, no signs of lumbosacral dural ectasia by MRI was seen in all four. So none of the four parents fulfilled the Ghent criteria for MFS even in the presence of an independently diagnosed child. A summary of the clinical features is shown in Table 1.

One grandparent (I.3, the father of the girl's father) had died at the age of 53 due to lung cancer.

\section{DNA analysis}

DNA was extracted from peripheral blood and analyzed by DHPLC and direct DNA sequencing as described previously. ${ }^{11}$

cDNA of the FBN1 gene was prepared from total RNA from cultured fibroblasts from a skin biopsy from the index patient. RNA was isolated using the RNA isolation minikit (Qiagen) according to the manufacturers instructions. Full-length single-stranded cDNA was prepared with oligodT-primer and Superscript ${ }^{\mathrm{TM}}$ II RT reverse trancriptase (Invitrogen). The complete coding sequence of the FBN1 gene was analyzed by direct sequencing in 24 overlapping PCR fragments (primer sequences available on request).

\section{Results and discussion}

The proband (III. 1) and his cousin (III.4), both with MFS, were homozygous for a mutation in exon 11 of the $F B N 1$ gene: c.1453C > T FBN1 mutation (p.Arg485Cys) (Figure 2). As expected, all four parents were heterozygous for the c.1453C > T FBN1 mutation. Of the siblings of the patients, 
Table 1 Summary of the clinical features in cases 1 and 2, and their parents ${ }^{\mathrm{a}}$

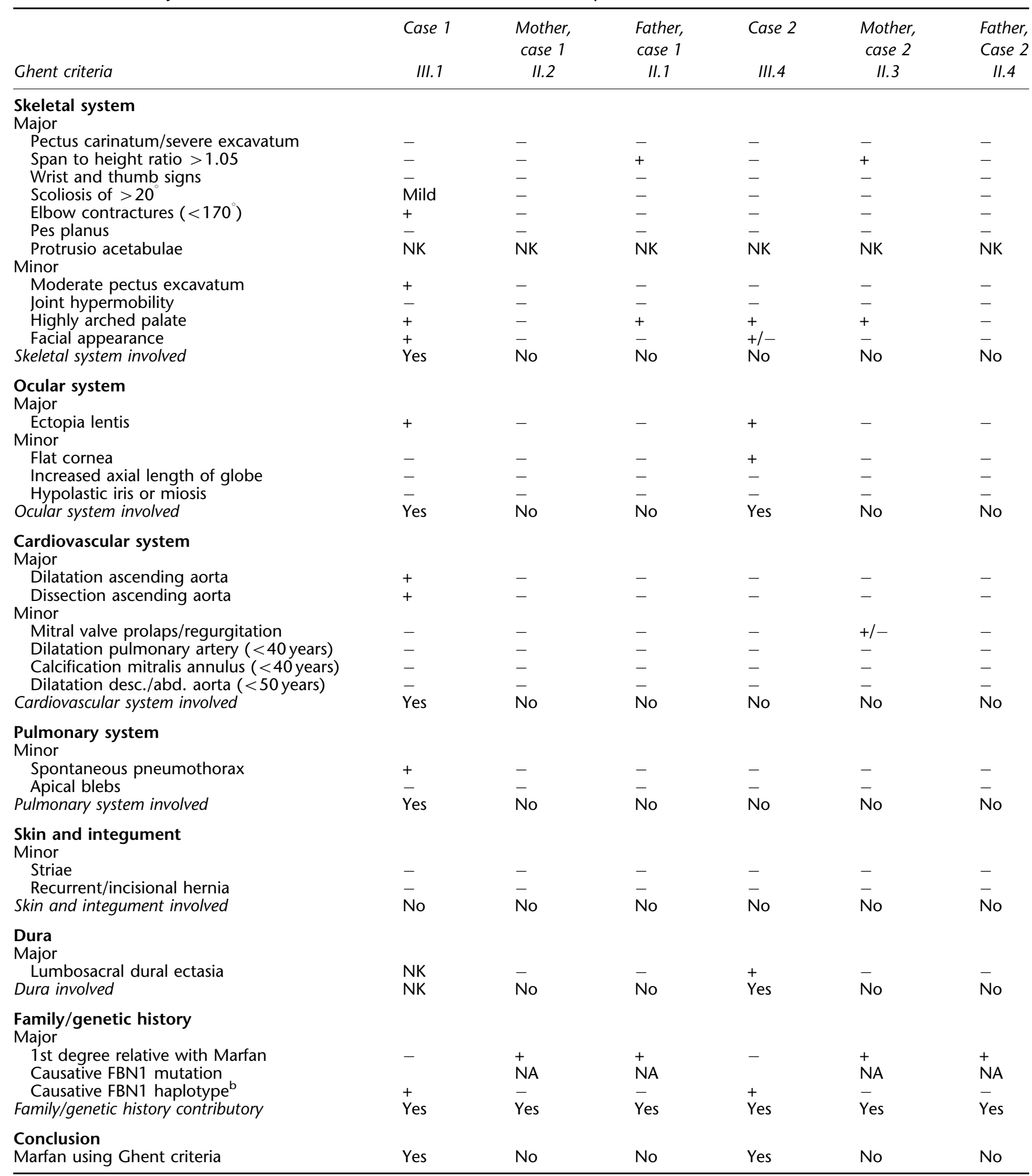

NA, not applicable; NK, not known.

${ }^{a}$ Numerical data for various characteristics are in the text.

${ }^{\mathrm{b}}$ Homozygous C.1453C > T FBN1 mutation. 


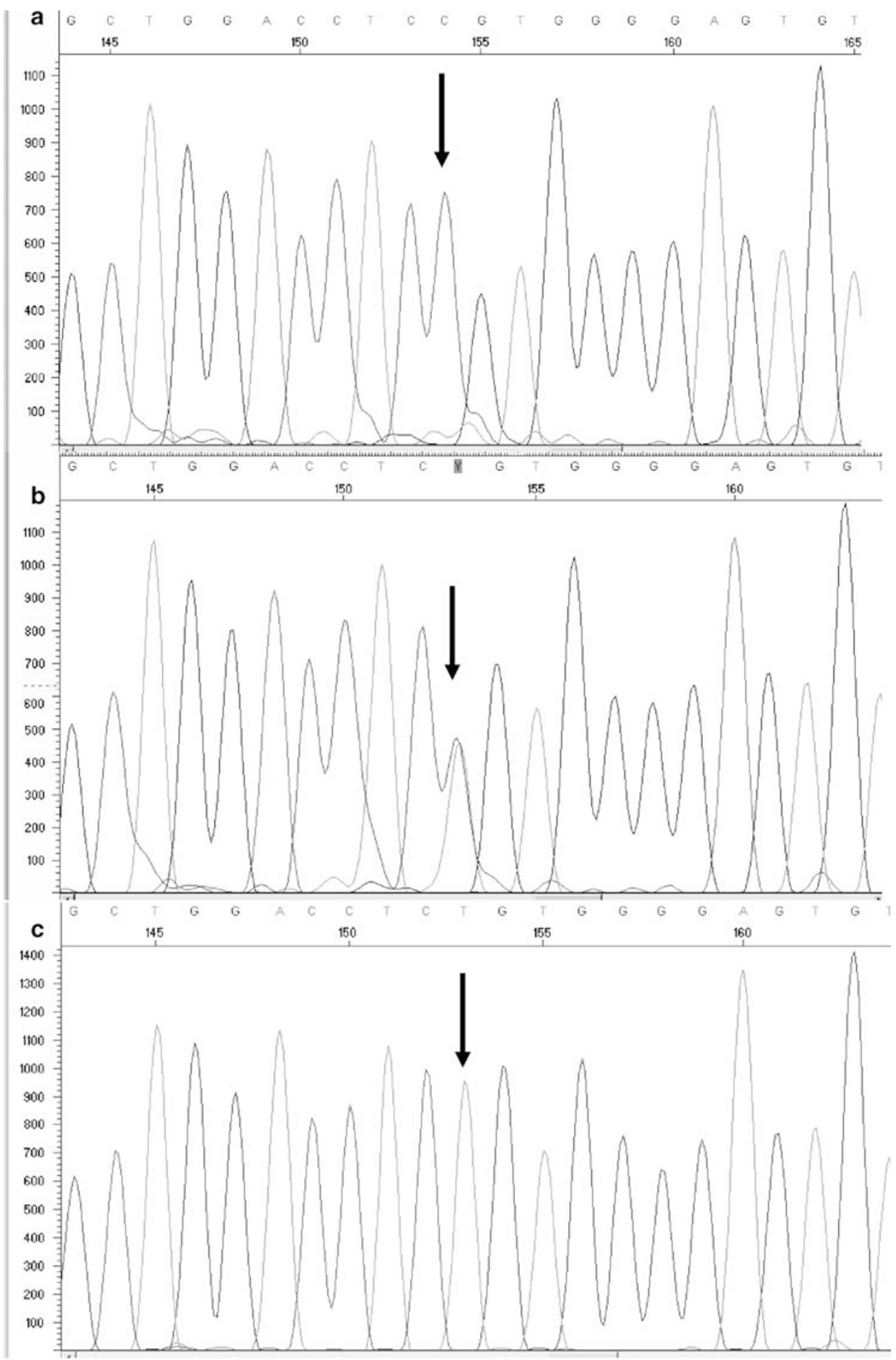

Figure 2 Mutation analysis in the FBN1 gene. (A) Wild type with $C$ at position 1453 in exon 11 (indicated by arrow). (B) Heterozygous C.1453C $>T$ mutation in exon 11, leading to the amino-acid substitution p.Arg485Cys as observed in normal parents. (C) Homozygous C.1453 $>T$ mutation in exon 11 as observed in both affected cousins. Sequence analysis was performed on DNA from peripheral blood on an ABI3730 sequencer, using BIGDYE version 3.2 chemistry (Applied Biosystems, Torrence, CA, USA), details available on request. 
only the 18-year-old sister (III.2) of the proband was tested and she was carrier of the mutation. She had no clinical features of MFS.

The mutation in exon 11 leads to the amino-acid substitution p.Arg485Cys. All 65 exons of the FBN1 gene were tested by DHPLC and only exon 11 showed an aberrant pattern and was analyzed by direct sequencing. Some homozygous mutations may be difficult to detect by DHPLC, but direct sequencing of the complete coding sequence of $F B N 1$ on cDNA from the proband showed no additional mutations. Moreover, there was no evidence of aberrant mRNA splicing. The Arg485Cys substitution has not been found previously and the mutation could not be detected in 500 control chromosomes. The substitution is located in a calcium-binding EGF-like domain, close to the sixth and last cysteine residue in this domain. The new cysteine residue is likely to disrupt the structure of the domain, because abnormal $\mathrm{S}=\mathrm{S}$ bridge formation will occur. This may lead to protein instability and inter- or intra-molecular crosslinks. The only mutation with a similar position within the EGF domain that has been described is $8038 \mathrm{C}>\mathrm{T}$, leading to Arg2680Cys. This mutation is in the C-terminal part of the protein and was found in a patient with MFS, with skeletal, ocular and pulmonary, but no cardiac involvement. ${ }^{12}$ In conclusion, if the Arg485Cys substitution had been found heterozygously in a patient with MFS or isolated ectopia lentis, we would have had no doubt about the pathogenic effect of the mutation.

Most of the disease-associated FBN1 mutations have been unique to one affected individual or family and are distributed throughout the gene. However, the most frequently observed mutations are missense mutations in the EGF motifs. Mutations introducing an extra cysteine within this motif, such as in our family, are likely to cause domain misfolding, which may lead a defective global structure and function of the protein. ${ }^{7}$ Clinically, this type of cysteine substitution mutations in a heterozygous state have been associated with a higher incidence of ectopia lentis compared to nonsense mutations leading to a premature stop codon. ${ }^{6,13}$

In the family we describe here, this mutation in heterozygous state did not cause the MFS. However, in homozygous state it caused a full-blown MFS in the 22year-old male and also for his 13-year-old cousin the Ghent criteria for MFS were met. Notably, in both cousins with the homozygous cysteine substitution mutation ectopia lentis could be observed at a rather young age of $\leq 3$ years. This is in line with the high incidence of ectopia lentis among MFS patients with similar, but heterozygous, missense mutations and confirms this previously noted genotype-phenotype relation. ${ }^{6,13}$ The observations that two cousins with a homozygous mutation are affected and all heterozygous parents are not affected, make it unlikely that an additional gene is involved in MFS in this family.
The current family is the first in which the recessive inheritance pattern of the MFS could be confirmed molecularly. Previously, some MFS cases supportive of recessive inheritance have been reported such as two girls from nonconsanguineous and unaffected parents by Fried and Krakowsky. ${ }^{14}$ However, as those clinical observations have not been molecularly confirmed other explanations such as gonadal mosaicism might still be a possibility.

MFS is inherited in an autosomal-dominant fashion in almost all previously reported families. The syndrome does not fulfill the classical requirement for 'true dominant' mutations that homozygotes do not differ clinically from heterozygotes as can be seen in disorders such as Huntington disease and myotonic dystrophy. ${ }^{15}$ Homozygous patients within MFS families have been reported and those were actually more severely affected than their family members with classical MFS, leading to severe fatal manifestations from birth. ${ }^{16-18}$ In the family reported by Schollin et al., ${ }^{18}$ Kartunnen et al. ${ }^{19}$ were able to show a compound heterozygosity in the lethally affected child: a Trp217Gly mutation from the father and a Gly2627Arg mutation from the mother. Both parents were affected although the mother in a lesser degree.

The current family might also shed some light on the pathogenesis of the MFS. For years, the dominant-negative model has been used to explain the occurrence of the clinical features in MFS. In this model, the mutant fibrillin might either hamper polymerization or lead to microfibrillar dysfunction. ${ }^{7}$ This concept has also been supported by mouse models. ${ }^{20}$ The dominant-negative model was challenged by the findings of Judge et al. ${ }^{21}$ that overexpression of mutant fibrillin actually failed to cause any abnormalities of cellular or clinical phenotype in mice leading to the conclusion that haploinsufficiency rather than production of mutant protein causes the phenotype.

The recessive inheritance in our family does not support the dominant-negative model, as the heterozygotes cannot be distinguished from wild type, but is more in line with a haploinsufficiency model. Different mechanisms may underlie the pathogenesis. Either in the homozygous cases not enough normal fibrils can be formed, due to protein degradation or intermolecular cross-linking, or the reduced levels of fibrillin might lead to an excess of active TGF- $\beta$, which is bound to fibrillin1 in an inactive form in the tissue. Reduced amounts of fibrillin may lead to excess amounts of active TGF- $\beta$. Excess of TGF- $\beta$ activity has been shown to be involved in the pathogenesis of MFS characteristics in mice deficient in fibrillin1. ${ }^{9,20}$ However, for the family we describe here alternative models cannot be excluded such as the formation of abnormal microfibrils due to abnormal fibrillin production.

In summary, this family is the first molecularly confirmed recessive MFS. The demonstration of recessive cases of MFS has obvious implications for genetic counselling as well as for molecular diagnosis. 


\section{Acknowledgements}

We thank the patients and their families for their kind cooperation and Dr Waelkens for referring the family and Dr FH Janssen for the MRI imaging in both families.

\section{Web Resources}

Online Mendelian Inheritance in Man (OMIM), http:// www.ncbi.nlm.nih.gov/Omim/.

\section{References}

1 Judge DP, Dietz HC: Marfan's syndrome. Lancet 2005; 366: $1965-1976$.

2 Dietz HC, Cutting GR, Pyeritz RE et al: Marfan syndrome caused by a recurrent de novo missense mutation in the fibrillin gene. Nature 1991; 352: 337-339.

3 De Paepe A, Devereux RB, Dietz HC, Hennekam RCM, Pyeritz RE: Revised diagnostic criteria for the Marfan syndrome. Am J Med Genet 1996; 62: 417-426.

4 Robinson PN, Godfrey M: The molecular genetics of Marfan syndrome and related microfibrillopathies. J Med Genet 2000; 37: $9-25$.

5 Robinson PN, Booms P, Katzke S et al: Mutations of FBN1 and genotype-phenotype correlations in Marfan syndrome and related fibrillinopathies. Hum Mutat 2002; 20: 153-161.

6 Loeys B, De Backer J, Van Acker P et al: Comprehensive molecular screening of the FBN1 gene favors locus homogeneity of classical Marfan syndrome. Hum Mutat 2004; 24: 140-146.

7 Robinson PN, Booms P: The molecular pathogenesis of the Marfan syndrome. Cell Mol Life Sci 2001; 58: 1698-1707.

8 Judge DP, Biery NJ, Keene DR et al: Evidence for a critical contribution of haploinsufficiency in the complex pathogenesis of Marfan syndrome. J Clin Invest 2004; 114: 172-181.

9 Neptune ER, Frischmeyer PA, Arking DE et al: Dysregulation of TGF-beta activation contributes to pathogenesis in Marfan syndrome. Nat Genet 2003; 33: 407-411.
10 Roman MJ, Devereux RB, Kramer-Fox R, O'Loughlin J: Twodimensional echocardiographic aortic root dimensions in normal children and adults. Am J Cardiol 1989; 64: 507-512.

11 Matyas G, De Paepe A, Halliday D, Boileau C, Pals G, Steinmann B: Evaluation and application of denaturing HPLC for mutation detection in Marfan syndrome: Identification of 20 novel mutations and two novel polymorphisms in the FBN1 gene. Hum Mutat 2002; 19: 443-456.

12 Palz M, Tiecke F, Booms P et al: Clustering of mutations associated with mild Marfan-like phenotypes in the 3 ' region of FBN1 suggests a potential genotype-phenotype correlation. Am J Med Genet 2000; 91: 212-221.

13 Schrijver I, Liu W, Odom $\mathrm{R}$ et al: Premature termination mutations in FBN1: distinct effects on differential allelic expression and on protein and clinical phenotypes. Am J Hum Genet 2002; 71: 223-237.

14 Fried K, Krakowsky D: Probable autosomal recessive Marfan syndrome. J Med Genet 1977; 14: 359-361.

15 Zlotogora J: Dominance and homozygosity. Am J Med Genet 1997; 68: $412-416$.

16 Capotorti L, Gaddini de Benedetti R, Rizzo P: Contribution to the study of the heredity of Marfan's syndrome: description of a family tree of 4 generations with marriage between consanguineous parents. Acta Genet Med Gemellol 1959; 8: 455-482.

17 Chemke J, Nisani R, Feigl A et al: Homozygosity for autosomal dominant Marfan syndrome. J Med Genet 1984; 21: 173-177.

18 Schollin J, Bjarke B, Gustavson K-H: Probable homozygotic form of the Marfan syndrome in a newborn child. Acta Paediat Scand 1988; 77: 452-456.

19 Karttunen L, Raghunath M, Lonnqvist L, Peltonen L: A compound-heterozygous Marfan patient: two defective fibrillin alleles result in a lethal phenotype. Am J Hum Genet 1994; 55: $1083-1091$.

20 Gayraud B, Keene DR, Sakai LY, Ramirez F: New insights into the assembly of extracellular microfibrils from the analysis of the Fibrillin 1 mutation in the tight skin mouse. J Cell Biol 2000; 150: $667-679$.

21 Judge DP, Biery NJ, Keene DR et al: Evidence for a critical contribution of haploinsufficiency in the complex pathogenesis of Marfan syndrome. J Clin Invest 2000; 114: 172-181. 